\title{
The Mouse formin (Fmn) Gene: Abundant Circular RNA Transcripts and Gene-Targeted Deletion Analysis
}

\author{
Cindy Wang Chao, David C. Chan, Ann Kuo, and Philip Leder
}

Department of Genetics and Howard Hughes Medical Institute, Harvard Medical School, Boston, Massachusetts, U.S.A.

Communicated by P. Leder. Accepted July 18, 1998.

\begin{abstract}
Background: Mutations in the mouse formin (Fmn) gene result in limb deformities and incompletely penetrant renal aplasia. A molecular genetic approach was taken to characterize novel circular RNAs from the Fmn gene and to understand the developmental effects of gene-targeted mutations.

Materials and Methods: RT-PCR and ribonuclease protection analyses were done to characterize the circular RNA transcripts arising from the Fmn gene. Two lines of mice with gene-targeted deletions of specific Fmn exons, namely exon 4 or exon 5, were generated and analyzed.

Results: In our analysis of formin cDNAs, we discovered a class of transcripts in which the exon order is reversed such that downstream exons are joined to the acceptor end of a specific exon that lies 5' to them in the genome. RT-PCR and ribonuclease protection analyses indicate that these transcripts are circular and are the major transcripts arising from this locus in adult brain and kidney.
\end{abstract}

To gain insight into the biological function of these transcripts, we have systematically deleted the relevant exons using gene-targeted homologous recombination. The resulting mice fail to produce circular transcripts, but appear to produce normal amounts of the linear RNA isoforms from this locus. While these deficient mice have normal limbs, they display variably penetrant renal aplasia characteristic of other mutant formin alleles.

Conclusions: Our results demonstrate novel circular transcripts arising from the Fmn gene. Moreover, their high levels of expression suggest that they are not products of aberrant splicing events, but instead, may play important biological roles. Mice with gene-targeted deletions of Fmn exons 4 or 5 lack these circular transcripts and have an incompletely penetrant renal agenesis phenotype. While the biologic function of circular Fmn RNA transcripts is not entirely known, our work suggests their possible involvement in kidney development.

\section{Introduction}

The mouse formin (Fmn) gene, formerly known as the limb deformity gene, is a large and com-

David C. Chan's present address is Department of Biology, Massachusetts Institute of Technology, and Howard Hughes Medical Institute, Whitehead Institute for Biomedical Research, 9 Cambridge Center, Cambridge, MA 02142.

Ann Kuo's present address is Department of Developmental Biology and Howard Hughes Medical Institute, Stanford University School of Medicine, Stanford, CA 94305.

Address correspondence and reprint requests to: Dr. Philip Leder, Department of Genetics and Howard Hughes Medical Institute, Harvard Medical School, 200 Longwood Avenue, Boston, Massachusetts 02115, U.S.A. Phone: (617) 432-7667; Fax: (617)432-7944 plex locus that spans 400 kilobases $(\mathrm{kb})$, contains 24 known exons, and encodes many alternatively spliced and differentially polyadenylated transcripts leading to multiple protein isoforms called formins $(1,2)$. Mice homozygous for $f m n$ mutations display an autosomal recessive, pleiotropic phenotype affecting the kidneys and limbs (3-8). The limb phenotype is completely penetrant and consists of oligodactyly, syndactyly, and fusion of the radius and ulna as well as tibia and fibula. At the embryonic level, mutations at this locus disrupt formation of the anteroposterior axis of the embryonic limb, and differentiation of the 
apical ectodermal ridge (AER) fails to occur properly in mutant fmn embryos (9-11).

The kidney phenotype is characterized by incompletely penetrant uni- or bilateral renal agenesis. Renal agenesis occurs at highly varying rates of incidence depending on the allele, ranging from $98 \%$ in $f m n^{l d-J}$ mutant animals to $2.5 \%$ in $f m n^{l d-I n 2}$ animals $(7,12)$. In addition to renal agenesis, $f m n^{l d-I n 2}$ mutant animals also display varying degrees of hydronephrosis and hydroureter (7). The morphologic basis of the renal agenesis phenotype in one of the fmn mutant alleles, $f m n^{l d-J}$, has been demonstrated to be due to delayed ureteric bud outgrowth (12). The differences in penetrance of the renal agenesis phenotype are likely due to strength differences inherent to the mutant alleles or to closely linked modifying loci rather than to differences in the genetic background (12). Recently, WynshawBoris et al. (13) have reported that homozygous fmn mutant animals with a targeted disruption of Fmn exon 6 have a $6 \%(6 / 105)$ incidence of unilateral or bilateral renal agenesis.

During the course of our studies of the murine formin locus, we discovered mRNA transcripts whose exons appeared to be assembled in an unexpected order. In eukaryotes, the splicing machinery converts preRNA into messenger RNA (mRNA), in which the $5^{\prime}$ to $3^{\prime}$ linear order of exons most often corresponds to their order in genomic DNA. In several cases, however, transcripts have been detected in which downstream exons are accurately spliced 5' of upstream exons. In eukaryotic genes, such splicing of a donor site of a downstream exon to the acceptor site of an upstream exon, known as exon scrambling, was first described in transcripts of the DCC (deleted in colorectal carcinoma) gene (14). To date, two other groups have reported scrambling of the genomic order of exons in eukaryotic RNA transcripts. In one case, novel transcripts of the human c-ets-1 gene were identified in which authentic splice sites were used, but the normal order of exons was reversed $(15,16)$. These scrambled transcripts are not polyadenylated and were found to be circular RNA molecules (17). In another case, involving the rat cytochrome P450 2 C24 gene, a circular RNA species was described in kidney and liver, which consisted of three exons with a scrambled exon junction (18). Circular transcripts of the testis-determining gene Sry in adult mouse testis have also been found, yet in this case only one exon is present in the circular RNAs, with the $5^{\prime}$ splice site of this single exon joined to its own $3^{\prime}$ splice site (19). None of these studies have addressed experimentally the question of the biological function of these circular RNA species.

In this study, we have identified novel circular RNAs of the Fmn gene from kidney and brain that contain scrambled exon junctions. To investigate the developmental role of specific Fmn exons and, in particular, the role of these circular RNA isoforms in the fmn phenotype, we generated two lines of mice deficient in either exon 4 or 5, exons that are present in all the scrambled transcripts identified thus far. Fmn exons 4 and 5 are expressed in adult brain and kidney and embryonic limb bud ectoderm $(1,20)$. Exon 4, which occupies a genomic position $5^{\prime}$ to most of the known Fmn coding exons, has termination codons in all three reading frames (1) such that translational products from a transcript containing exon 4 would be prematurely terminated. Homozygous mutants in both of these gene-targeted mouse lines, called $f m n^{4 K O}$ and $f m n^{5 K O}$, exhibit the fmn mutant kidney phenotype of incompletely penetrant renal agenesis and furthermore, lack Fmn circular transcripts. Their limb formation, however, is entirely normal.

\section{Materials and Methods}

Subcloning DNA Fragments, Southern Blot Transfer, Labeling/Hybridization of Probes, and RNA Expression Analysis

Subcloning, Southern blot transfer, and ${ }^{32} \mathrm{P}-\mathrm{la}-$ beling and hybridization of DNA probes were done using standard procedures (21). Ribonuclease protection assays were done as described previously (22).

\section{Construction and Screening of cDNA Libraries}

cDNA libraries from adult murine brain and kidney poly $(A)+$ RNA were constructed using the lambda ZAP bacteriophage vector and reagents from Stratagene according to the manufacturer's instructions. For each RNA source (brain or kidney), two libraries were made, using either random hexamer primers or an $\mathrm{Fmn}$-specific primer (from the exon 5-exon 7 junction) to prime the cDNA. The oligonucleotide sequence for this exon 5-exon 7 primer is as follows: $5^{\prime}$ tctggtcagggttgagatcc $3^{\prime}$. Oligo $\mathrm{d}(\mathrm{T})$ priming was not used to improve the chances of finding $5^{\prime}$ sequences of the Fmn gene and novel exons $5^{\prime}$ of exon 4. Filters with $\sim 1 \times 10^{6}$ clones from each of the four unamplified libraries were hybridized 
to a ${ }^{32} \mathrm{P}$-radiolabeled $85-\mathrm{nt}$ oligonucleotide of the exon 4 sequence. The sequence of this oligonucleotide is the sequence of the entire DNA sense strand of Fmn exon 4 (1). Positive clones were isolated and sequenced.

\section{RT-PCR Assay to Assess for Circular Transcripts}

First-strand cDNA was synthesized according to the manufacturer's instructions, using reagents and Superscript reverse transcriptase from Gibco BRL. CDNA was primed using an oligonucleotide primer from within Fmn exon 4 (primer A: 5' caaccattcaaatga $3^{\prime}$ ) from $5 \mu \mathrm{g}$ of adult, wild-type murine brain or kidney total RNA. The sequences of the oligonucleotide polymerase chain reaction (PCR) primers that face outward from exon 4 are as follows: primer Bl: $5^{\prime}$ acctacaacatcagcctgg 3'; primer B2: 5' gaaaagcacagactttcaaag $3^{\prime}$. Standard PCR conditions (Perkin Elmer) were used. Negative control PCR reactions were set up using the same brain and kidney cDNA samples and $5^{\prime}$-terminal and $3^{\prime}$-terminal exon 4 primers that faced toward each other (i.e., faced inward). With these primers $\left(5^{\prime}\right.$ cccaggctgatgttgtagg $3^{\prime}$ and $5^{\prime}$ ctttgaaagtctgtgctt $\left.3^{\prime}\right)$, a PCR product would not be amplified using a cDNA template that has been primed from within the middle of exon 4; furthermore, an exon 4 PCR product would not be detected with the Fmn exon 5 oligonucleotide probe shown in Figure 3B. The negative control result from the brain cDNA is shown in Figure 3B; the results from the kidney cDNA were the same (data not shown). The PCR products were gel electrophoresed and then transferred by Southern blotting to GeneScreen (NEN). The filter was subsequently hybridized with an Fmn exon 5 oligonucleotide ( $5^{\prime}$ cgtctgctctttgaagttgtcacaggg $\left.3^{\prime}\right)$. Exon 5-hybridizing bands were subcloned into the $\mathrm{pCR}^{\mathrm{TM}} \mathrm{II}$ vector (Invitrogen) prior to sequencing.

When this assay was repeated on RNA samples from the brain or kidney of Fmn exon 5-deleted mice and control animals, the same exon 4 oligonucleotide as described above (primer A) was used as a cDNA primer. The outward-facing exon 4 PCR primers that were used in this $f m n^{5 K O}$ analysis are as follows: $5^{\prime}$ gagctgcaccttgttgatgc $3^{\prime}$ and $5^{\prime}$ gaaaagcacagactttcaaag $3^{\prime}$ (same as primer B2, shown above). For the control RT-PCR to test for the presence of cDNA in each sample, the PCR primers are from exon $2\left(5^{\prime}\right.$ gagaccaaaggagccagccc $\left.3^{\prime}\right)$ and exon $4\left(5^{\prime}\right.$ gagctgcaccttgttgatgc $\left.3^{\prime}\right)$. The RT-PCR products were hybridized with a $5^{\prime}$-terminal exon 4 oligonucleotide $\left(5^{\prime}\right.$ ccccaggctgatgttgtagg $3^{\prime}$ ).

For $f m n^{4 K O}$ and control animals (wild-type, heterozygous mutant, and homozygous mutant), cDNA was primed with an oligonucleotide primer from within Fmn exon 5 ( $5^{\prime}$ agggtggtccggctctgtaac $\left.3^{\prime}\right)$. The sequences of the oligonucleotide PCR primers that face outward from exon 5 are as follows: $5^{\prime}$ ctgctccatgaagagaaagagaag $3^{\prime}$ and $5^{\prime}$ catccttattgggaaggtctt $3^{\prime}$. The RT-PCR assay was done as described above, and the Southern blot filter was hybridized with a $5^{\prime}$-terminal exon 5 oligonucleotide $\left(5^{\prime}\right.$ gtattgctctgaaggcttt $\left.3^{\prime}\right)$. For the control samples, the PCR primers were from exon 2 (same as in the control for the $f m n^{5 K O}$ analysis) and exon 5 ( $5^{\prime}$ catccttattgggaaggtctt $\left.3^{\prime}\right)$.

\section{Targeting Vectors}

The 129/Sv genomic clones containing Fmn exons 4 and 5 and flanking sequences were isolated from a lambda FIX II library (Stratagene) using an exon 4 probe generated by PCR, using a $5^{\prime}$ oligonucleotide $\left(5^{\prime}\right.$ ccccaggctgatgttgtagg $\left.3^{\prime}\right)$ and a $3^{\prime}$ oligonucleotide $\left(5^{\prime}\right.$ ctttgaaagtctgtgctt $\left.3^{\prime}\right)$. To construct the $f m n^{4 K O}$ targeting vector, we subcloned a $3.5 \mathrm{~kb} E c o \mathrm{RV}-E c o \mathrm{RV}$ genomic fragment upstream from exon 4 and a $4.8 \mathrm{~kb} E c o R I-E c o R I$ genomic fragment downstream of exon 4 into the vector pPNT (22), kindly provided by V. Tybulewicz. To construct the $f m n^{5 K O}$ targeting vector, we subcloned a $4.5 \mathrm{~kb}$ Bam HI-EcoRI genomic fragment upstream from exon 5 and a $3 \mathrm{~kb}$ PstI$X b a \mathrm{I}$ genomic fragment downstream of exon 5 into the pPNT vector (23).

Homologous Recombination in Embryonic Stem Cells and Generation of Germline Chimeras

TCl embryonic stem (ES) cells derived from a 129/SvEvTacfBR mouse (24) were transfected separately with NotI-digested $f m n^{4 K O}$ and NotIdigested $f m n^{5 K O}$ targeting vector DNA and selected with G41 8 and FIAU. The culture, electroporation, and selection of ES cells were carried out as described previously (25). To identify clones having undergone homologous recombination events, genomic DNAs from the $f m n^{4 K O}$ clones and the parental TCl cell line were digested with XbaI, followed by Southern blot transfer analysis using a $0.3 \mathrm{~kb}$ PCR probe $5^{\prime}$ to the targeting vector (Fig. 4C). The oligonucleotides for this probe are as follows: $5^{\prime}$ cacacatacacatcatgtgg $3^{\prime}$ and $5^{\prime}$ tcctgccttctaacatgttc $3^{\prime}$. To identify homologously recombined $f m n^{5 K O}$ 
clones, genomic DNAs from the G418 and FIAUselected clones and the parental TCl cell line were digested with Bam HI and ClaI, followed by Southern blot analysis using a $0.8 \mathrm{~kb}$ probe $3^{\prime}$ to the targeting vector (see Fig. $4 \mathrm{C}$ ). Because this probe contains some repetitive sequences, the Southern blot results were reconfirmed with various other sets of restriction enzymes and alternative probes within the targeting vector (data not shown). ES cells heterozygous for the targeted mutations $\left(f m n^{4 K O}\right.$ or $f m n^{5 K O}$ ) were microinjected into $\mathrm{C} 57 \mathrm{BL} / 6 \mathrm{~J}$ blastocysts, and germline chimeras were generated as previously described (26). Chimeric males were mated with NIH Black Swiss (Taconic) female mice to test for germline transmission (25), and F1 animals heterozygous for the targeted mutations were intercrossed to generate all genotypes.

\section{Protein Analysis}

To prepare total organ protein lysates, dissected brain or kidney organs were placed in 5 volumes of lysis buffer and homogenized with a Polytron (Brinkmann Instruments) tissue blender as previously described (27). An equal volume of $2 \times$ SDS-polyacrylamide gel electrophoresis (SDS-PAGE) sample buffer was added, and the samples were heated in a boiling water bath for $5 \mathrm{~min}$. The samples were sonicated (Sonifier, Branson) to reduce their viscosity and reboiled before being subjected to SDS-PAGE on a $6 \%$ gel. Western blots were performed using standard procedures (28) with antisera A and B (27), which recognize epitopes encoded by Fmn exons 9 and 6, respectively. Protein bands were visualized by enhanced chemoluminescence (ECL) (Amersham).

\section{Results}

\section{Identification of Novel Fmn cDNA Clones}

Our previous studies showed that transcripts containing Fmn exon 4 are expressed in embryonic tissues as well as in adult tissues such as brain and kidney (1). To retrieve these exon 4-containing Fmn cDNAs, we screened adult murine brain and kidney cDNA libraries with a ${ }^{32}$ P-labeled Fmn exon 4 probe. Six major classes of transcripts containing exon 4 were obtained. In Figure 1, the top diagram shows the arrangement of Fmn exons in the murine genome with the cDNAs containing exon 4 shown below. Sur- prisingly, 5 of these cDNAs represent scrambled transcripts (14), since exons found upstream of exon 4 in these cDNAs are actually located downstream of exon 4 in the genome.

To rule out the possibility of cDNA cloning artifacts and to confirm that these scrambled transcripts are authentic Fmn transcripts, a ribonuclease protection assay was done with ribonuclease protection probes (riboprobes) that span all of the scrambled exon junctions shown in Figure 1, as well as with a riboprobe for the nonscrambled cDNA clone containing exon 4 (shown as the first cDNA clone in Fig. 1). Representative results of assays using three ribonuclease protection probes are shown in Figure 2. Strikingly, these scrambled transcript-specific riboprobes detected strong expression in adult brain and kidney. The scrambled transcripts were found to be highly abundant, representing up to $70-80 \%$ of the formin RNA detected by the riboprobe. Each of these scrambled transcripts, all of which exhibit the scrambled junction at the acceptor site $5^{\prime}$ of exon 4, were more abundant than the nonscrambled exon 4-containing transcript. Similar levels of abundance in brain and kidney were found in ribonuclease protection assays with the riboprobes for the other scrambled junctions (data not shown).

\section{Evidence for Circularity of Fmn RNA Transcripts}

When scrambled transcripts were first described, it was hypothesized that such transcripts were likely to be circular in structure (14). Subsequent inverse PCR studies on the scrambled transcripts of the c-ets-1 and cytochrome P450 2C24 genes have provided strong evidence for this model $(17,18)$. We devised a similar inverse PCR assay to test whether the scrambled Fmn transcripts were circular. We synthesized first-strand cDNA from wild-type brain and kidney RNA using an exon 4 primer, denoted primer $A$, and performed PCR with flanking oligonucleotide primers, denoted B1 and B2, that anneal to exon 4 sequences and face outward (see Fig. 3A). With normal linear transcripts, PCR with these outward-facing primers would not yield a discrete product; only circular RNAs would yield specific amplification products. Indeed, PCR amplification resulted in 402-bp and 340-bp RT-PCR products (Fig. 3B) that hybridized with the downstream exon 5 probe. Subcloning and sequence analysis showed that these RT-PCR products probably represent two circular RNA species, which are shown schematically in Figure $3 \mathrm{C}$ 


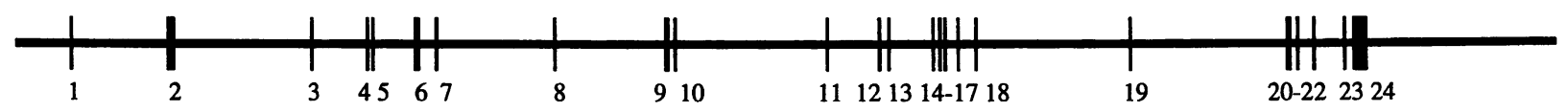

$-=10 \mathrm{~kb}$

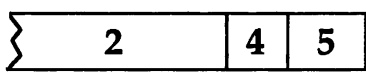

\begin{tabular}{|l|l|l|l|l|||l|l|}
\hline 5 & 7 & 8 & 9 & 10 & 4 & 5 \\
\hline
\end{tabular}

\begin{tabular}{|l|l|l|l|l|||l|l|}
\hline 7 & 8 & 9 & 10 & 11 & 4 & 5 \\
\hline
\end{tabular}

\begin{tabular}{|l|l|l|l|l|l|l|l|||l|l|}
\hline 7 & 8 & 9 & 10 & 11 & 12 & 13 & 4 & 5 \\
\hline
\end{tabular}

18||

4

\section{5}

5

\begin{tabular}{|l|l|}
\hline 9 & $10-16$ \\
\hline
\end{tabular}

Fig. 1. Novel Fmn cDNA clones representing scrambled transcripts. At the top, the $F m n$ genomic structure (1) is depicted with the exons numbered as shown. Below are six classes of cDNA clones isolated with an exon 4 probe by screening murine adult brain and kidney cDNA libraries (made by priming with random hexamers and a Fmn exon

with the locations of the PCR primers indicated. One circular species contains Fmn exons 4, 5, 7, and 8; the other species contains exons 4, 5, and 7. In the former, a scrambled exon junction occurs between exons 8 and 4, while in the latter, a scrambled exon junction occurs between exons 7 and 4 . The exons were joined accurately at their consensus splice sites (data not shown).

We repeated the ribonuclease protection assays of the scrambled Fmn transcripts using total RNA, poly(A) + RNA, and non-poly(A) + RNA from adult murine brain and kidney. These scrambled transcripts are not retained in a poly $(\mathrm{A})+$ fractionation column, but were present in the non-poly(A) + flow-through fractions (data not shown), suggesting that they do not contain a poly $(\mathrm{A})+$ tail; this is similar to previous reports of circular transcripts $(14,16,17)$. 5/7-junction primer). The numbers in the cDNA clone diagrams represent sequences from the corresponding Fmn exon. A scrambled exon junction, where a downstream exon is spliced $5^{\prime}$ to an upstream exon, is indicated by a thick black bar between two thin white bars.

\section{Targeted Deletions of Exons 4 and 5 of the Fmn Gene}

Since all the scrambled cDNAs isolated contained exons 4 and 5, we decided to analyze the in vivo function of these exons and their corresponding circular transcripts by designing the gene-targeting constructs $f m n^{4 K O}$ (Fig. $4 \mathrm{~A}$ ) and $f m n^{5 K O}$ (Fig. 4B) to delete exons 4 and 5, respectively. The fmn ${ }^{4 K O}$ construct contains $8.8 \mathrm{~kb}$ of $\mathrm{Fmn}$ genomic sequence with exon 4 replaced by the phosphoglycerate kinase-neomycin resistance gene (abbreviated (PGK)neo) cassette from the pPNT vector (23). The $f m n^{5 K O}$ construct contains $7.5 \mathrm{~kb}$ of $\mathrm{Fmn}$ genomic sequence with exon 5 replaced by the same (PGK) neo cassette. Homologous recombination within the Fmn locus employing these targeting constructs would introduce the neomycin resistance gene (neo) and delete Fmn exon 4 or 5, depending on the con- 


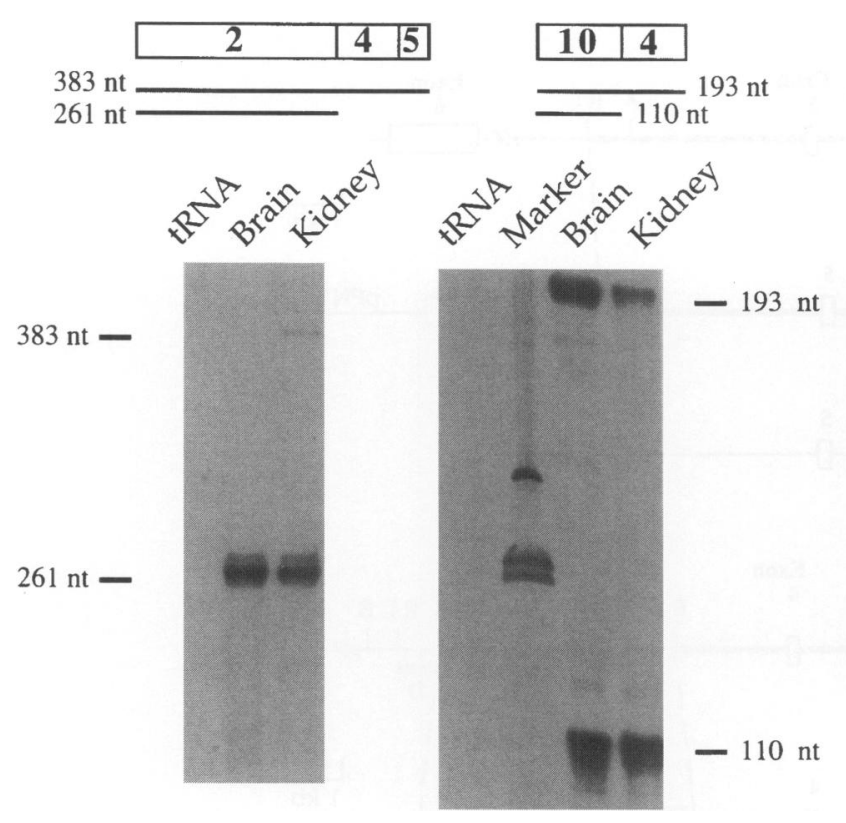

struct used. Of 200 G418 and FIAU double-resistant embryonic stem (ES) cell clones that were screened by Southern blot analysis for homologous recombination with the $f m n^{4 K O}$ construct, two had undergone a correct targeting event (Fig. 4C). Germline transmission was obtained from the injection of one of these ES clones (clone \#23) into C57BL/6J blastocysts. For the $f m n^{5 K O}$ construct, five of $100 \mathrm{G} 418$ and FIAU double-resistant ES cell clones were positive for the correct targeting event (one example is shown in Fig. 4C). Two of these positive ES clones (clones \#6 and \#15), heterozygous for the targeted mutation, were injected into C57BL/6J blastocysts, and germline transmission was obtained for both of these clones. For both genetargeted mutants $f m n^{4 K O}$ and $f m n^{5 K O}$, mice het-
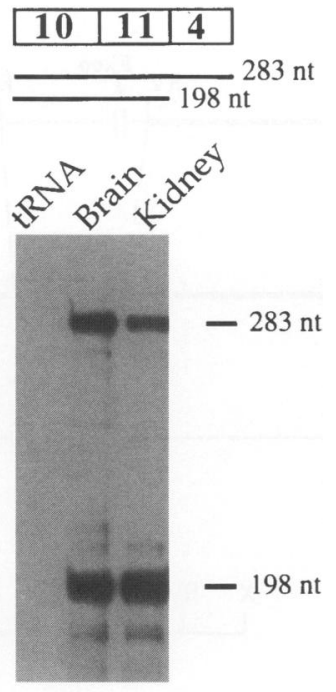

Fig. 2. Fmn scrambled transcripts are abundant, authentic transcripts. A ribonuclease protection assay is shown of adult brain and kidney total RNA (tRNA) with riboprobes to detect normal and scrambled transcripts containing exon 4 The numbers in the riboprobe diagrams represent the Fmn exon sequences contained in the riboprobe. The normal-order riboprobe contains exons 2, 4, and 5 sequences. The scrambled-order riboprobes contain exon 10-exon 4 and exon 10exon 11-exon 4 sequences.

erozygous for the mutation were intercrossed to obtain wild-type, heterozygous mutant, and homozygous mutant animals. Figure $4 \mathrm{D}$, a Southern blot of DNA samples from wild-type, heterozygous mutant, and homozygous mutant $f m n^{4 K O}$ and $f m n^{5 K O}$ mice, demonstrates that the Fmn exon 4 and exon 5 DNA sequences are absent from the respective homozygous mutant $f m n^{4 K O}$ and $f m n^{5 K O}$ animals.

\section{Expression Analysis of Mice Deleted for Either Fmn Exon 4 or Exon 5}

Ribonuclease protection assays were used to assess the specificity of the gene-targeted deletions and to confirm that the expression of other Fmn isoforms in either brain or kidney is not affected.

Fig. 3. Evidence of circularity of Fmn RNA transcripts. An RT-PCR assay was used to assess the circularity of Fmn transcripts. (A) The relative locations of the primers used in this RT-PCR are shown in the schematic diagram of Fmn exon 4. Primer A was used to synthesize first-strand cDNA from wild-type murine adult brain and kidney RNA. The cDNA was amplified with the PCR primers $\mathrm{B} 1$ and $\mathrm{B} 2$, which face outward from exon 4 . (B) Southern blot of RT-PCR products from brain and kidney cDNA and a negative control (described in Materials and Methods) hybridized with an exon 5 probe. (C) Schematic diagram of two circular RNA species represented by the subcloned and sequenced 402-bp and 340-bp RT-PCR products from this assay. Arrows represent the PCR primers B1 and B2, used in this assay. Numbers represent the complete Fmn exons, which were joined accurately at their consensus splice sites. 


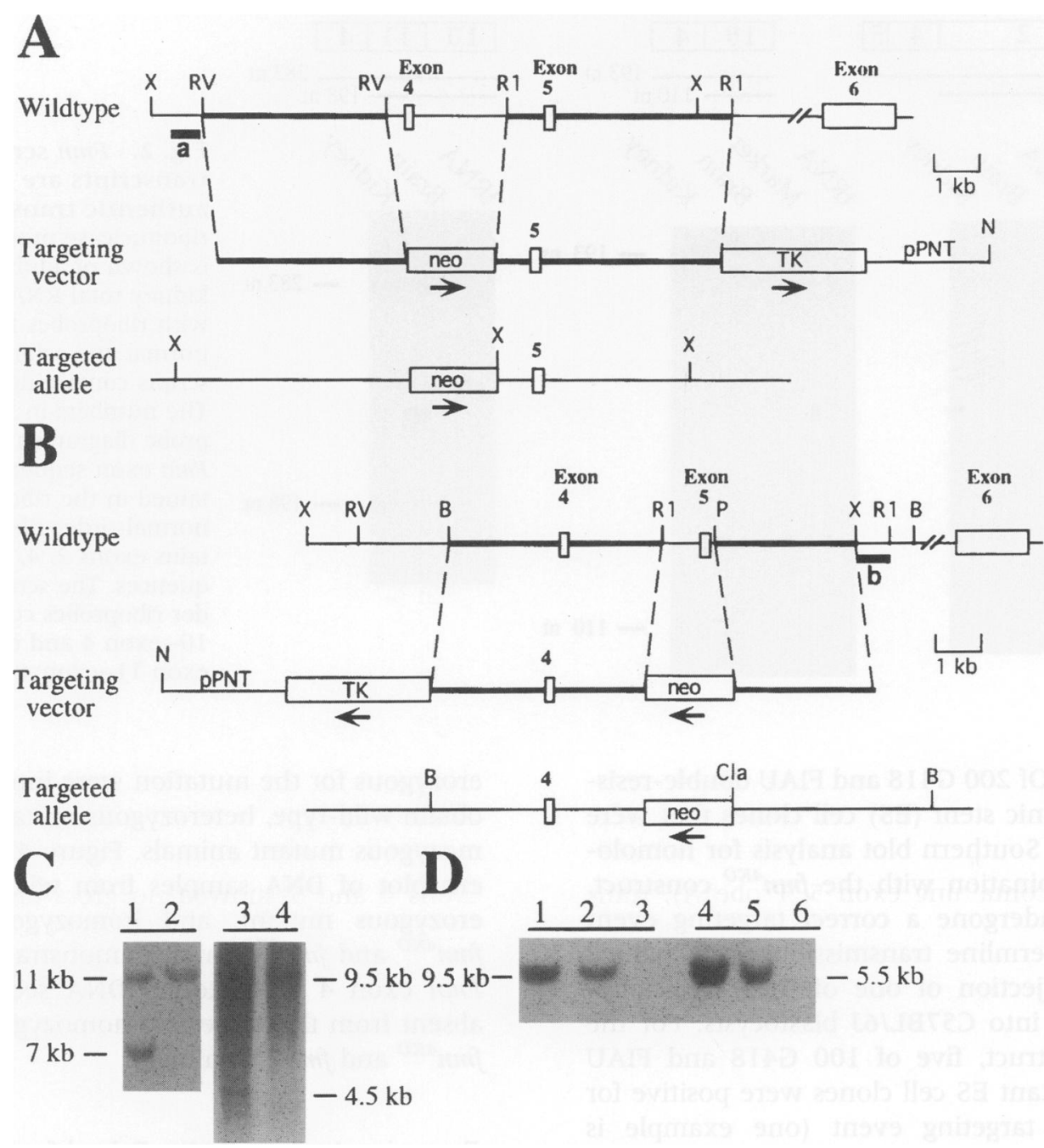

Fig. 4. Fmn exon-specific gene-targeting approach for separately deleting exons 4 and 5 . The positions of a neomycin resistance gene (neo) replacing the respective deleted $F m n$ exon as well as a herpes simplex virus thymidine kinase gene $(T K)$ for positive/negative selection from the pPNT vector (23) are indicated. Arrows indicate the transcriptional directions of the neo and TK genes. Prior to electroporation, the targeting vectors were linearized at the indicated unique NotI (N) sites. B, BamHI; Cla, ClaI; P, PstI; RI, EcoRI; RV, EcoRV; X, XbaI. (A) Gene-targeting Fmn exon 4-deletion construct. The replacement-type targeting construct contains $3.5 \mathrm{~kb}$ of genomic DNA upstream of exon 4 and $4.8 \mathrm{~kb}$ of downstream homologous sequence. Homologous recombination within the Fmn locus would introduce the neo gene and delete 2.5 kb of genomic DNA containing Fmn exon 4. (B) Genetargeting Fmn exon 5-deletion construct. The replacement-type targeting construct contains $4.5 \mathrm{~kb}$ of genomic DNA upstream of exon 5 and $3 \mathrm{~kb}$ of downstream homologous sequence. Homologous recombination within the Fmn locus would introduce the neo gene and delete $1.1 \mathrm{~kb}$ of genomic DNA containing Fmn exon 5. (C) Southern blot analysis for targeted exon 4- and exon 5-deleted ES cell clones. DNA was isolated from targeted ES cell clones (lanes 1 and 3) and parental ES cells (lanes 2 and 4). As expected, the $X b a I$ restriction fragment size change from $11 \mathrm{~kb}$ to 7 $\mathrm{kb}$ is seen in the targeted exon 4 -deleted ES cell clone (lane 1) using the $0.3 \mathrm{~kb} 5^{\prime}$ outside probe (a) indicated in Fig. 4A. Similarly, the BamHI/ClaI restriction fragment size change from $9.5 \mathrm{~kb}$ to $4.5 \mathrm{~kb}$ is seen in the targeted exon 5-deleted ES cell clone (lane 3) using the $0.8 \mathrm{~kb}^{\prime}$ outside probe (b) indicated in Fig. 4B. (D) Homozygous Fmn exon 4- and exon 5-deletion mice lack DNA sequences for exons 4 and 5, respectively. DNA isolated from wild-type, heterozygous mutant, and homozygous mutant littermate animals were first genotyped by Southern blot analysis. Then the same DNA samples were hybridized with a probe specific for the deleted Fmn exon. Wild-type, heterozygous $f m n^{4 K O}$, and homozygous $f m n^{4 K O}$ DNA samples (lanes 1, 2, and 3, respectively) digested with BamHI were hybridized with a Fmn exon 4 probe. As indicated in the wild-type allele diagram in Fig. $4 \mathrm{~B}$, a $9.5 \mathrm{~kb}$ BamHI-digested DNA fragment is detected using a Fmn exon 4 probe. Wild-type, heterozygous $f m n^{5 K O}$, and homozygous $f m n^{5 K O}$ DNA samples (lanes 4, 5, and 6, respectively) digested with EcoRI were hybridized with a Fmn exon 5 probe. As shown in the wild-type allele diagrams in Fig. $4 \mathrm{~A}$ and B, a Fmn exon 5 probe detects a $5.5 \mathrm{~kb}$ EcoRI-digested DNA fragment. 


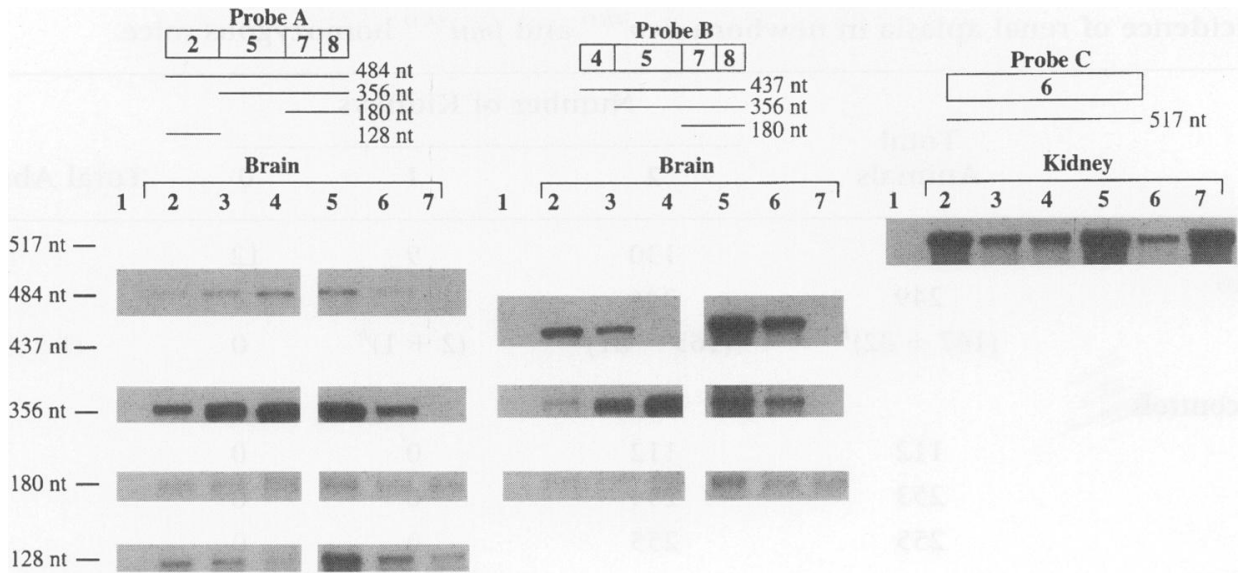

Fig. 5. RNA expression analysis in Fmn exon 4- and exon 5-deficient mice. Shown is a ribonuclease protection assay using a riboprobe specific for Fmn isoform I (probe A), a riboprobe containing Fmn exon 4 (probe $\mathrm{B}$ ), and a riboprobe specific for Fmn isoform IV (probe C) on RNA samples from the following sources: 1 , yeast tRNA control; 2 , wildtype $(+/+)$ littermate of animals 3 and $4 ; 3$,

We used riboprobes that detected Fmn isoform I transcripts (containing exon 5; Probe A), transcripts containing exon 4 and 5 (Probe B), and isoform IV transcripts (containing exon 6; Probe C) (Fig. 5), (1). As expected, expression of exons 4 and 5 is absent in the respective homozygous mutant samples. For the $f m n^{4 K O} /+$ and $f m n^{4 K O}$ / $f m n^{4 K O}$ samples, the isoform I transcripts, indicated by the 484-nt protected species, and isoform IV transcripts, indicated by the 517-nt protected species, are present and grossly unaffected compared to wild-type RNAs. For the $f m n^{5 K O} / f m n^{5 K O}$ brain sample, a fully protected isoform I 484-nt band is not observed since exon 5 is present in isoform I. However, expression of Fmn exon 2, which is spliced $5^{\prime}$ to exon 5 in isoform I transcripts, is still present and grossly unaffected, as represented by the 128-nt protected species. Similar to the $f m n^{4 K O}$ samples, in $f m n^{5 K O} /+$ and $f m n^{5 K O} / f m n^{5 K O}$ kidney samples, isoform IV transcripts (517-nt protected species) were present and grossly unaffected. We used an L32 ribosomal RNA riboprobe as a loading control (data not shown) and found that normalized levels for isoform I and IV were quantitatively similar in all genotypes. Therefore, these results indicate that while $f m n^{4 K O}$ and $f m n^{5 K O}$ homozygous mutants show no expression of the deleted exons, other Fmn isoforms are present at grossly normal levels. Similarly, Western blot analyses with two anti-formin antibodies recognizing $\mathrm{fmn}^{4 \mathrm{KO}} /+; 4, \mathrm{fmn}^{4 \mathrm{KO}} / \mathrm{fmn} \mathrm{n}^{4 \mathrm{KO}} ; 5,+/+$ littermate of animals 6 and $7 ; 6, f m n^{5 K O} /+; 7, f m n^{5 K O} / f m n^{5 K O}$. The organ (brain or kidney) of the murine adult RNA samples (lanes 2-7) is indicated above the lane numbers. The numbers in the riboprobe diagrams represent the Fmn exon sequences contained in the riboprobe.

epitopes encoded by Fmn exons downstream of exons 4 and 5 showed no gross differences in formin protein expression in kidney lysates from all genotypes of $f m n^{4 K O}$ and $f m n^{5 K O}$ mice (data not shown).

Renal Aplasia Is Observed in $\mathrm{fm}^{4 \mathrm{KO}}$ and $\mathrm{fm}^{5 \mathrm{KO}}$ Animals

Mice heterozygous for the targeted mutations $\left(f m n^{4 K O} /+\right.$ and $\left.f m n^{5 K O} /+\right)$ were developmentally and anatomically normal. F2 offspring for both targeted mutations were generated by the intercrossing of $\mathrm{Fl} f m n^{4 K O} /+$ or $f m n^{5 K O} /+$ mice. At weaning, mice homozygous for either targeted mutation $\left(f m n^{4 K O} / f m n^{4 K O}\right.$ or $\left.f m n^{5 K O} / f m n^{5 K O}\right)$ were present at the expected frequencies according to Mendelian patterns of inheritance. No limb abnormalities were found in any heterozygous or homozygous $f m n^{4 K O}$ or $f m n^{5 K O}$ mutant mice, and they were developmentally normal and fertile. Large-scale matings among $f \mathrm{mn}^{4 \mathrm{KO}}$, $f m n^{4 K O}$ homozygous mutant animals and matings among $f m n^{5 \mathrm{KO}} / \mathrm{fmn}^{5 \mathrm{KO}}$ animals were done to score for low-penetrance mutant phenotypes, such as renal agenesis, in the newborn progeny. Animals were sacrificed and dissected within the first $24 \mathrm{hr}$ after birth since animals with bilateral renal aplasia die shortly after birth. As shown in Table 1, fm ${ }^{4 K O} / f m n^{4 K O}$ homozygous mutant animals were observed to have uni- and bilateral 
Table 1. Incidence of renal aplasia in newborn $f m n^{4 K o}$ and $f m n^{5 K O}$ homozygous mice

\begin{tabular}{|c|c|c|c|c|c|}
\hline \multirow[b]{2}{*}{ Genotype $^{a}$} & \multirow{2}{*}{$\begin{array}{c}\text { Total } \\
\text { Animals }\end{array}$} & \multicolumn{3}{|c|}{ Number of Kidneys } & \multirow[b]{2}{*}{ Total Abnormal (\%) } \\
\hline & & 2 & $\mathbf{1}$ & $\mathbf{0}$ & \\
\hline$f m n^{4 K O} / f m n^{4 K O}$ & 151 & 130 & 9 & 12 & 13.9 \\
\hline$f m n^{5 K O} / f m n^{5 K O}$ & $\begin{array}{c}249 \\
(167+82)^{b}\end{array}$ & $\begin{array}{c}246 \\
(165+81)^{b}\end{array}$ & $\begin{array}{c}3 \\
(2+1)^{b}\end{array}$ & $\begin{array}{l}0 \\
0\end{array}$ & $\begin{array}{c}1.2 \\
(1.2 \text { and } 1.2)^{b}\end{array}$ \\
\hline $\begin{array}{l}\text { Background cor } \\
\mathrm{fm}^{4 \mathrm{KO} /+}\end{array}$ & 112 & 112 & 0 & 0 & 0 \\
\hline $\mathrm{fmn}^{5 \mathrm{KO}} /+$ & 253 & 253 & 0 & 0 & 0 \\
\hline$+1+$ & 255 & 255 & 0 & 0 & 0 \\
\hline
\end{tabular}

${ }^{a}$ The listed genotypes were derived from matings of homozygous animals. Newborns were sacrificed within $24 \mathrm{hr}$ of birth.

${ }^{b}$ Numbers in parentheses are the breakdown data from two $f m n^{5 K O} / f m n^{5 K O}$ mouse lines derived from two independently derived targeted ES cell clones (clones 6 and 15, respectively).

renal agenesis at an incidence rate of $13.9 \%$ $(21 / 151)$, and $f m n^{5 K O} / f m n^{5 K O}$ animals had an incidence rate of unilateral renal agenesis of $1.2 \%$ $(3 / 249)$. Examples of this renal agenesis phenotype are shown in the photographs in Figure 6. Besides complete renal agenesis, no other renal abnormalities were detected using histologic sections of kidneys from heterozygous and homozygous $f m n^{4 K O}$ and $f m n^{5 K O}$ mice. Both adrenal glands (see Fig. 6) and the urinary bladder were always present. No gross defects in the male or female genital structures were observed.

To address the formal possibility that the low level $(1.2 \%)$ of renal agenesis observed in $f m n^{5 K O} / \mathrm{fmn}^{5 \mathrm{KO}}$ animals could reflect the background degree of renal agenesis seen in mice with this genetic background [combination of 129/SvEv (inbred) and NIH Black Swiss (outbred)], matings of homozygous animals from the same mixed genetic background were also done to score newborn $f m n^{5 K O} /+$ heterozygous mice and wild-type mice for renal abnormalities. In this assay, $253 \mathrm{fmn}^{5 \mathrm{KO}} /+$ mice and $255+1+$ mice were sacrificed at birth to check for renal anomalies, and none were found among all 508 of these mice (Table 1). Similarly, $112 \mathrm{fm}^{4 K O} /+$ newborn mice also had no renal abnormalities (Table 1). Using the chi-square statistical test with one degree of freedom to compare the data from our $f m n^{5 K O} / f m n^{5 K O}$ experimental set (3 animals with renal agenesis and 246 unaffected animals) and a control set consisting of the $f m n^{5 K O} /+$ and $+I+$ mice (0 animals with renal agenesis and 508 unaffected animals with a similar genetic background to the experimental set of animals), we find that $\chi^{2}=3.97$, and $p<0.05$. It should also be noted that our observed $1.2 \%$ incidence of renal agenesis in $f m n^{5 K O} / \mathrm{fmn}^{5 \mathrm{KO}}$ animals was observed in lines derived from two independently derived ES cell lines.

In addition, homozygous $f m n^{4 K O} / \mathrm{fmn}^{4 K O}$ and $f m n^{5 K O} / f m n^{5 K O}$ mice were mated to other $\mathrm{fmn}$ homozygous mutants to create compound heterozygotes to test for noncomplementation of the fmn mutant kidney phenotype (see Table 2). We detected noncomplementarity between the $f m n^{4 K O}$ and $f m n^{T g B r i 137}$ alleles with a $12 \%$ incidence of renal agenesis (Table 2). The incidence of renal aplasia, if any, in the other matings may require larger numbers of offspring to detect. In summary, mice homozygous for the Fmn exon 4 deletion or exon 5 deletion have normal limbs and an incompletely penetrant renal aplasia phenotype. This suggests that the deletion of either Fmn exon 4 or 5 is sufficient for the renal agenesis phenotype but not the limb-deformity phenotype seen in fmn mutant mice.

\section{Circular Fmn Transcripts Are Not Detected in} $\mathrm{fmn}^{4 \mathrm{KO}}$ and $\mathrm{fmn}^{5 \mathrm{KO}}$ Mutant Mice

Since Fmn exons 4 and 5 are present in all of the scrambled and circular Fmn transcripts that have been detected thus far, we repeated the RT-PCR assay described previously (see Fig. 3) to assess for the presence of Fmn circular transcripts in 

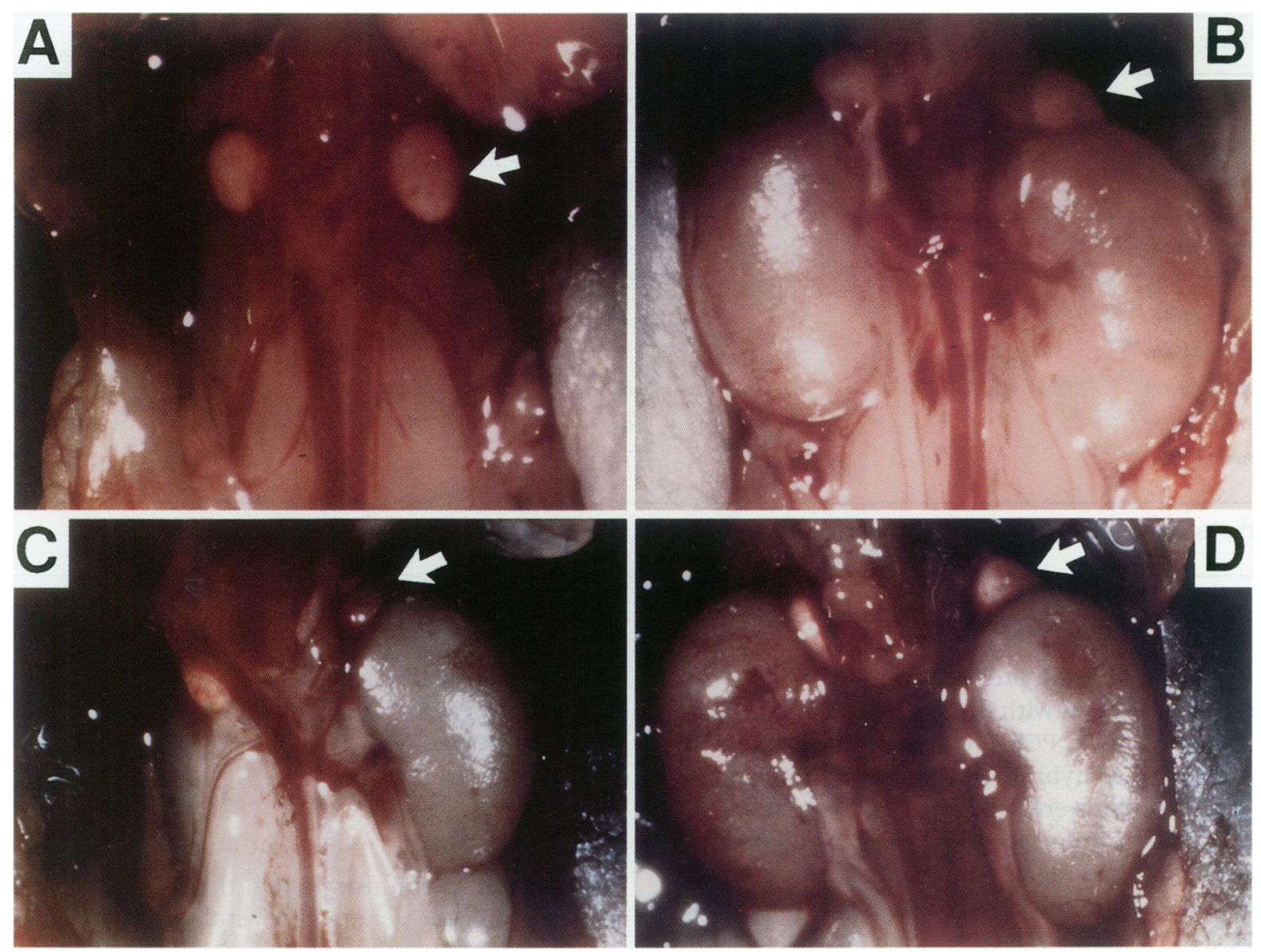

Fig. 6. Bilateral and unilateral renal agenesis in Fmn exon 4- and exon 5-deficient mice. These photographs of the retroperitoneal area of neonatal $f m n^{4 K O} / f m n^{4 K O}$ and $f m n^{5 K O} / f m n^{5 K O}$ animals illustrate the bilateral and unilateral renal agenesis phenotype seen in these mice at the incidence rates presented in Table 1. Organs contained within the peritoneum that obscured the view of the kidney region were removed prior to photographing. The

organs of our $f m n^{4 K O}$ and $f m n^{5 K O}$ mice. We used RNA from adult brain and kidney since these organs express high levels of circular RNA. For the analysis of $f m n^{4 K O}$ samples, first-strand cDNA was primed with a Fmn exon 5 primer from brain and kidney RNA of mice with the following genotypes: $+l+$ (littermate of the $f m n^{4 K O}$ mutant animals), $f m n^{4 K O} /+, f m n^{4 K O} / f m n^{4 K O}$ (Fig. 7A). Note that an exon 5 primer, not exon 4 primer as in Figure 3, has been used in this assay since the homozygous $f m n^{4 K O} / f m n^{4 K O}$ animal has been deleted for the entire exon 4. PCR amplification was performed using flanking primers facing outward from exon 5. RT-PCR products were hybridized with an exon 5 oligonucleotide probe,

adrenal glands (at level of white arrows), which are located near the top pole of the kidney, were always present bilaterally. (A) $f m n^{4 K O} / f m n^{4 K O}$ animal with bilateral renal agenesis. (B) $f m n^{4 K O} / f m n^{4 K O}$ littermate animal with normal two kidneys (for comparison). (C) $f m n^{5 K O} / \mathrm{fm}^{5 K O}$ animal with unilateral renal agenesis. (D) $f m n^{5 K O} / f m n^{5 K O}$ littermate animal with normal two kidneys (for comparison).

subcloned, and sequenced. As shown in Figure 7A (lane 3 for both brain and kidney samples), no circular Fmn transcripts were detected in the homozygous $f m n^{4 K O} / f m n^{4 K O}$ brain or kidney. The 395-bp and 333-bp PCR products found in the wild-type and heterozygous $f m n^{4 K O} /+$ animals were sequenced, and they represent two circular RNA species (Fig. 7C) that are the same as those in Figure 3C. As in Figure 3, the Fmn exons in these circular transcripts were joined accurately at their consensus splice sites.

We simultaneously performed control PCR reactions to ensure that cDNA was present in each sample. For the control PCR (Fig. 7A), we used primers from exons 2 and 5, which are able 
Table 2. Incidence of renal aplasia in newborn fmn compound heterozygous mutant mice

\begin{tabular}{|c|c|c|c|c|c|}
\hline \multirow[b]{2}{*}{ Genotype $^{a}$} & \multirow[b]{2}{*}{$\mathbf{N}$} & \multirow[b]{2}{*}{2} & \multicolumn{3}{|c|}{ Number of Kidneys } \\
\hline & & & 1 & $\mathbf{0}$ & $\begin{array}{c}\text { Total } \\
\text { Abnormal (\%) }\end{array}$ \\
\hline$f m n^{4 K O} / f m n^{l d-T g B r i l 37}$ & 50 & 44 & 2 & 4 & 12 \\
\hline$f m n^{4 K O} / f m n^{l d-I n 2}$ & 60 & 60 & 0 & 0 & 0 \\
\hline$f m n^{4 K O} / f m n^{l d-G K O}$ & 44 & 44 & 0 & 0 & 0 \\
\hline$f m n^{4 K O} / f m n^{5 K O}$ & 52 & 52 & 0 & 0 & 0 \\
\hline$f m n^{5 K o} / f m n^{l d-T g B r i 137}$ & 48 & 48 & 0 & 0 & 0 \\
\hline$f m n^{5 K O} / f m n^{l d-I n 2}$ & 68 & 68 & 0 & 0 & 0 \\
\hline$f m n^{5 K O} / f m n^{l d-G K O}$ & 47 & 47 & 0 & 0 & 0 \\
\hline
\end{tabular}

${ }^{a}$ The listed genotypes were derived from matings of homozygous animals. Newborns were sacrificed within $24 \mathrm{hr}$ of birth.

to amplify a PCR product from a linear Fmn transcript primed with the same exon 5 primer as described above. PCR products from the control reactions that hybridized to an exon 5 oligonucleotide probe were subcloned and sequenced to confirm that the appropriate exon 2 and exon 5 sequences had been amplified. The expected control PCR products were amplified from all of the $f m n^{4 K O}$ cDNA samples; the brain cDNA control samples are shown in Figure 7A.

This circular RNA RT-PCR assay was done similarly to analyze $f m n^{5 K O}$ mice. Since the homozygous $f m n^{5 K O} / f m n^{5 K O}$ mice lack Fmn exon 5 (see Fig. 4D), oligonucleotide primers from exon 4, similar to those in the original circular RNA assay (Fig. 3), were used. cDNA was synthesized with an exon 4 primer using brain and kidney RNAs of $+/+, f m n^{5 K O} /+$, and $f m n^{5 K O} / f m n^{5 K O}$ animals. PCR amplification was performed using primers that faced outward from exon 4 (Fig. 7B). The control PCR reactions used primers from exons 2 and 4. As shown in Figure 7B (lane 3 for both brain and kidney samples), no circular Fmn transcripts were detected in the homozygous $f m n^{5 K O} / f m n^{5 K O}$ brain or kidney, as was the case with the homozygous $f m n^{4 K O} / f m n^{4 K O}$ animal. The 424-bp and 362-bp PCR products found in the wild-type and heterozygous $\mathrm{fmn}^{5 \mathrm{KO}} /+$ animals, which were sequenced, represent the same two circular RNA species as in Figure 3 and the above $f m n^{4 K O}$ analysis (Fig. 7C). The exons of these circular species were also joined accurately at their consensus splice sites. The appropriate control PCR products were amplified from all of the $f m n^{5 K O}$ cDNA samples; the brain cDNA control samples are shown in Figure 7B. An additional smaller band was also seen in the control samples (Fig. 7B), and this PCR product was not sequenced.

\section{Discussion}

Our results demonstrate that the large and complex Fmn gene generates several scrambled transcripts that likely have a circular structure. Moreover, their high levels of expression suggest that they are not products of aberrant splicing events, but instead, may play important biological roles. Some of the scrambled transcripts are expressed in the embryonic limb (1) and kidney (data not shown), suggesting that these unusual transcripts may play a role in the fmn mutant phenotype. To test this, we created gene-targeted deletions of two such exons, Fmn exons 4 and 5 . We wished to investigate the function of these exons in kidney and limb development as well as to examine the possible in vivo function of circular RNAs. We were able to recapitulate the incompletely penetrant fmn kidney phenotype with our $f m n^{4 K O}$ and $f m n^{5 K O}$ mice and to separate the $f m n$ kidney phenotype from the limb phenotype. The $f m n^{4 K O}$ and $f m n^{5 K O}$ homozygous mice do not produce detectable levels of circular transcripts, suggesting a possible role of these circular RNAs in the renal agenesis phenotype.

\section{Comparison to Other Reported Cases of Scrambled Transcripts}

As shown in Figure 2, the scrambled Fmn transcripts identified in this study are authentic tran- 

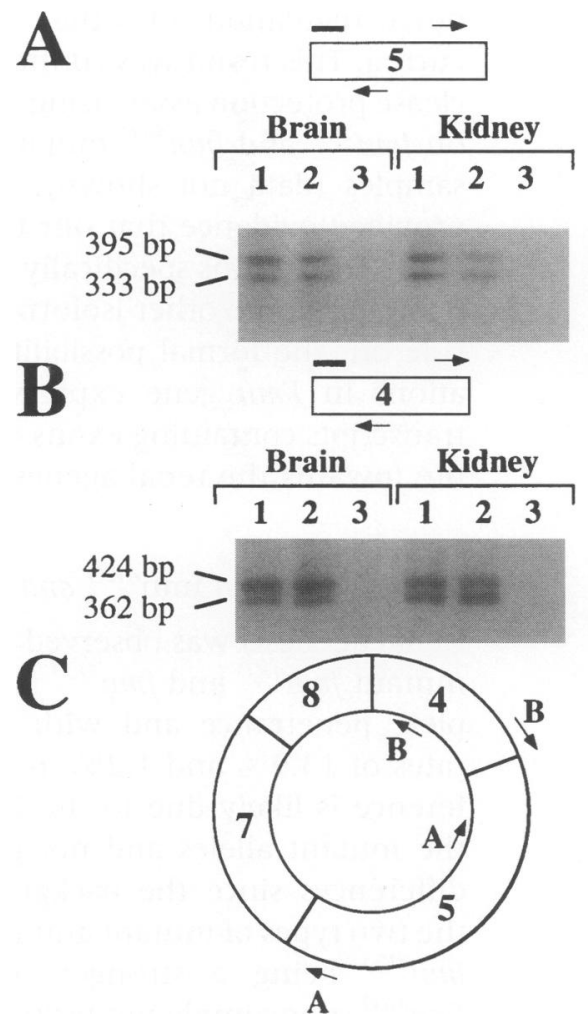

Fig. 7. Circular Fmn transcripts are not detected in the brain and kidney of Fmn exon 4and exon 5-deficient mice. An RT-PCR assay (see Fig. 3) was used to identify circular Fmn transcripts from organs of $f m n^{4 K O}$ and $f m n^{5 K O}$ mice. The locations of the PCR primers used in this RT-PCR assay are shown in the schematic diagrams in parts $\mathrm{A}$ and $B$. The numbers represent the Fmn exon sequences amplified in the PCR reactions. The black bars represent the locations of oligonucleotide probes for Southern blot hybridization of the PCR products shown below the schematic diagram. For this assay, positive controls (Control, A and B), which were PCR amplification of a normal, linear Fmn transcript, show that cDNA was present in each sample. The locations of the PCR primers for these controls are shown in the schematic diagram. The control lanes shown represent RT-PCR products from brain RNA samples. (A) RT-PCR analysis of $f m n^{4 K O}$ samples. First-strand cDNA, primed with a Fmn exon 5 primer, was synthesized from adult brain and kidney

scripts and represent up to $70-80 \%$ of the transcripts detected in kidney and brain by the riboprobe. This abundance is in striking contrast to other genes with scrambled or circular transcripts. For example, the DCC gene scrambled transcripts were present at $1 / 1000$ th of the concentration of normally spliced transcripts (14). Moreover, attempts to identify cDNA clones with scrambled exon junctions from cDNA libraries were unsuccessful in the case of $D C C$. Similarly,

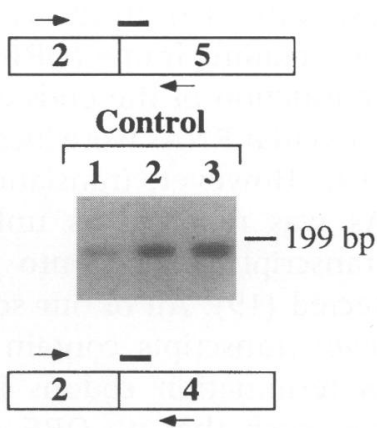

$$
\begin{array}{|l|l|}
\hline \multicolumn{3}{|c}{\text { Control }} \\
\hline \begin{array}{lll}
1 & 2 & 3
\end{array}
\end{array}
$$
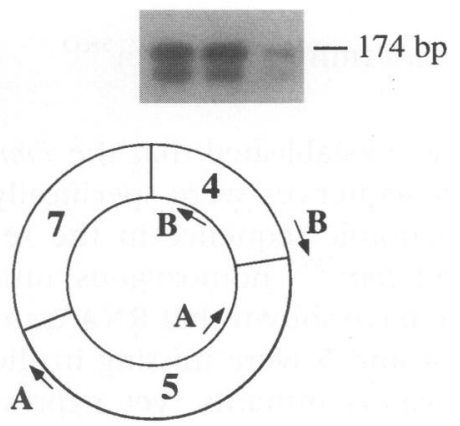

from mice with the following genotypes: $1,+1+$ littermate of animals 2 and 3; $2, f m n^{4 K O} /+; 3, f m n^{4 K O} /$ $f m n^{4 K O}$. RT-PCR products were hybridized with an exon 5 oligonucleotide probe. (B) RT-PCR analysis of $f m n^{5 K O}$ samples. First-strand cDNA, primed with a Fmn exon 4 primer, was synthesized from adult brain and kidney from mice with the following genotypes: $1,+1+$ littermate of animals 2 and 3; 2 , $f m n^{5 K O} /+; 3, \mathrm{fm}^{5 \mathrm{KO}} / \mathrm{fm} n^{5 \mathrm{KO}}$. RT-PCR products were hybridized with an exon 4 oligonucleotide probe.

(C) A schematic diagram of two circular RNA species (same as in Fig. 3C) represented by the subcloned and sequenced RT-PCR products from the wild-type and heterozygous $f m n^{4 K O}$ and $f m n^{5 K O}$ animals in this assay. Arrows labeled A represent the outward-facing exon 5 PCR primers shown in part A, which were used to analyze the $f m n^{4 K O}$ mice. Arrows labeled $B$ represent the similar primers shown in part B. Numbers represent the complete Fmn exons, which were joined accurately at their consensus splice sites.

the level of the scrambled human c-ets-1 transcripts were estimated to be only $1 \%$ of the level of the normally spliced transcripts (15). The high levels of scrambled Fmn transcripts suggest that they are not simply due to missplicing events but rather, play an important biological function.

Similar to abundant circular transcripts of Fmn, the single-exon circular transcript of the Sry gene has been shown by ribonuclease protection assays to represent up to $90 \%$ of the Sry tran- 
scripts in adult mouse testis (19). In this circular Sry transcript, an open-reading frame (ORF) was preserved across the junction of the ends of the single exon, and the circular RNAs were localized to the cytoplasm (19). However, translation of these circular RNAs was reported as unlikely since no circular transcripts loaded onto polysomes could be detected (19). All of our scrambled and circular Fmn transcripts contain Fmn exon 4, which has termination codons in all three reading frames, such that no ORF exists across the scrambled exon junction.

\section{Specificity of the Targeted $\mathrm{fm}^{4 \mathrm{KO}}$ and $\mathrm{fm}^{5 \mathrm{KO}}$ Mutations}

In this study, we have established that the Fmn exon 4 and exon 5 sequences were specifically deleted from the genomic sequence in the respective $f m n^{4 K O}$ and $f m n^{5 K O}$ homozygous mutants. Moreover, we have shown that RNA transcripts from exons 4 and 5 were missing in the appropriate homozygous mutants, yet expression of other exons, such as exon 2 , which is present in Fmn isoforms I-III, and exon 6, which is the amino-terminal exon of isoform IV, was relatively unaffected. Mice with homozygous targeted disruptions of exon $6, f m n^{l d-G K O}$, display a renal agenesis phenotype with a $6 \%$ incidence (13). Hence, it is important to note that our targeted mutations have not perturbed isoform IV (an exon 6-containing isoform) expression, as shown by our ribonuclease protection and Western blot assays. Accordingly, we can rule out the simple explanation that the renal agenesis phenotype seen in our targeted mutant mice arises secondarily to alterations in Fmn isoform IV expression. Our laboratory has also recently generated mice with a targeted disruption of exon 2, an exon near the amino-terminal region of isoforms I-III, which are expressed in the embryonic dorsal root ganglia, cranial ganglia, and the developing kidney (10). To date, two homozygous mutant $f m n^{2 \mathrm{KO}} / f m n^{2 \mathrm{KO}}$ adult animals have been observed to have unilateral renal agenesis (Y. Wang, personal communication). Thus, our RNA expression analyses of exon 2, whose level of expression is unaffected in our $f m n^{4 K O}$ and $f m n^{5 K O}$ mutants, are similarly important to rule out the possibility that perturbations in isoforms I-III cause the renal agenesis phenotype. Our immunoblot analysis with antisera recognizing epitopes encoded by Fmn exon 9, which is present in all of the coding linear Fmn isoforms, indicated that no overall up-regulation or down-regulation of other Fmn isoforms occurred. This result was confirmed with a ribonuclease protection assay using an exon 9 riboprobe on $f m n^{4 K O}$ and $f m n^{5 K O}$ mutant and control RNA samples (data not shown). Although we have provided evidence that our targeted $f m n^{4 K O}$ and $f m n^{5 K O}$ mutations specifically disrupt exons 4 and 5 and leave the other isoforms intact, we cannot rule out the formal possibility that subtle alterations in Fmn gene expression, beyond those transcripts containing exons 4 or 5 , may contribute towards the renal agenesis phenotype.

Renal Agenesis in $\mathrm{fm}^{4 \mathrm{KO}}$ and $\mathrm{fmn}^{5 \mathrm{KO}}$ Animals

Renal agenesis was observed in our homozygous mutant $f m n^{4 K O}$ and $f m n^{5 K O}$ mice with an incomplete penetrance and with different incidence rates of $13.9 \%$ and $1.2 \%$, respectively. This difference is likely due to the intrinsic strength of the mutant alleles and not genetic background differences since the background is similar for the two types of mutant animals. Consistent with $f m n^{4 K O}$ being a stronger mutant allele than $f m n^{5 K O}$, noncomplementarity of the renal agenesis phenotype between $f m n^{4 K O}$ or $f m n^{5 K O}$ and other $f m n$ mutant alleles was only observed with $f m n^{4 K O}$ and not with $f m n^{5 K O}$ in combination with another fmn mutant allele. This difference in strength may be related to the fact that exon 4 is a "terminator" exon. Transcripts that contain exon 4 will have an early termination in the translation product, and perhaps its presence plays an important negative regulatory role for translation in normal kidney development. More interestingly, all of the scrambled exon junctions that we have identified in this study always have exon 4 at the scrambled junction spliced to various downstream exons. Thus, although exon 5 is present in all of our characterized scrambled/ circular transcripts, exon 4 may play a much more critical role in the circular RNA formation, which, in turn, may be related to the more severe renal agenesis phenotype seen in $f m n^{4 K O}$, $f m n^{4 K O}$ mice. We also cannot rule out the possibility that the disruption of the linear (normal order) Fmn transcripts that contain either exon 4 or exon 5 are responsible for or contribute to the renal agenesis phenotype.

\section{Biological Function of Circular RNAs}

We have created gene-targeted mutations of Fmn exons 4 and 5 with the goal of achieving a better understanding of the fmn mutant phenotype as 
well as to investigate the potential in vivo function of circular Fmn transcripts. The observation that our gene-targeted mutant mice develop incompletely penetrant renal agenesis and also lack circular Fmn transcripts suggests a link between these circular transcripts and kidney development. The molecular function of circular Fmn RNA transcripts is of course not known, but we propose a model in which circular Fmn RNAs act as a functional mRNA "trap." In this model, all unprocessed Fmn RNAs that become spliced to form a circular RNA molecule become nonfunctional RNAs in that they are not translated to produce functional formin protein products. In our case, this model is consistent with Fmn circular RNAs not being translated; the circular Fmn transcripts all contain exon 4 , which has termination codons in all three reading frames. Thus, the formation of circular RNAs traps the transcripts arising from the Fmn gene in a nonfunctional form and prevents the existence of certain normal linear transcripts that could be translated. The mechanisms controlling circular RNA formation could thus regulate the expression levels of the normal linear RNA transcripts and thereby regulate the gene function. In a simple regulation model, higher levels of expression of circular RNA would correlate with lower levels of normal linear transcripts. The great abundance of circular forms relative to linear forms is consistent with such a regulatory role. It could also be possible that the relationship between circular RNA formation and normal linear transcripts production is modulated by other factors, making the relationship between circular and linear RNA less straightforward. In our study, circular RNA formation may perhaps be involved in renal organogenesis. Circular RNAs may later be discovered to have an even broader function in the regulation of many other biological functions. We hope that further characterization of the circular Fmn transcripts will yield answers to some of these questions.

\section{Acknowledgments}

We thank Mark Bedford and Chuxia Deng for their technical expertise and helpful discussions. We gratefully acknowledge technical help from Cathie Daugherty, Judy Dunmore, Anne Harrington, and Montserrat Michelman. We are grateful to Hsiuchen Chen, Ian Krane, Ben Stanger, and Christoph Westphal for careful reading of the manuscript. C. W. Chao and D. C.
Chan were supported in part by the NIH Medical Scientist Training Program.

\section{References}

1. Wang CC, Chan DC, Leder P (1997) The mouse formin (Fmn) gene: genomic structure, novel exons, and genetic mapping. Genomics 39: 303-311.

2. Woychik RP, Maas RL, Zeller R, Vogt TF, Leder $P$ (1990) 'Formins': proteins deduced from the alternative transcripts of the limb deformity gene. Nature 346: 850-853.

3. Cupp MB (1960) Limb-deformity, ld. Mouse News Lett. 22: 50.

4. Green MC, Masiak C (1962) Limb deformity. Mouse News Lett. 26: 34-35.

5. Rhodes R (1994) Remutation at limb deformity locus. Mouse Genome 92: 355.

6. Woychik RP, Stewart TA, Davis LG, D'Eustachio P, Leder $P$ (1985) An inherited limb deformity created by insertional mutagenesis in a transgenic mouse. Nature 318: 36-40.

7. Woychik RP, Generoso WM, Russel LB, et al. (1990) Molecular and genetic characterization of a radiation-induced structural rearrangement in mouse chromosome 2 causing mutations at the limb deformity and agouti loci. Proc. Natl. Acad. Sci. U.S.A. 87: 2588-2592.

8. Messing A, Behringer RR, Slapak JR, Lemke G, Palmiter RD, Brinster RL (1990) Insertional mutation at the ld locus (again!) in a line of transgenic mice. Mouse Genome 1990: 107.

9. Zeller R, Jackson-Grusby L, Leder P (1989) The limb deformity gene is required for apical ectodermal ridge differentiation and anteroposterior limb pattern formation. Genes Dev. 3: 1481-1492.

10. Chan DC, Wynshaw-Boris A, Leder P (1995) Formin isoforms are differentially expressed in the mouse embryo and are required for normal expression of $f g f-4$ and $s h$ in the limb bud. Development 121: 3151-3162.

11. Haramis AG, Brown JM, Zeller R (1995) The limb deformity mutation disrupts the SHH/FGF-4 feedback loop and regulation of $5^{\prime}$ HoxD genes during limb pattern formation. Development 121: 42374245.

12. Maas R, Elfering S, Glaser T, Jepeal L (1994) Deficient outgrowth of the ureteric bud underlies the renal agenesis phenotype in mice manifesting the limb deformity (ld) mutation. Dev. Dyn. 199: 214228.

13. Wynshaw-Boris A, Ryan G, Deng C-X, et al. (1997) The role of a single formin isoform in the limb and renal phenotypes of limb deformity. Mol. Med. 3: 372-384.

14. Nigro JM, Cho KR, Fearon ER, et al. (1991) Scrambled exons. Cell 64: 607-613.

15. Cocquerelle C, Daubersies $P$, Majérus M-A, Kerckaert J-P, Bailleul B (1992) Splicing with inverted 
order of exons occurs proximal to large introns. EMBO J. 11: 1095-1098.

16. Cocquerelle C, Mascrez B, Hétuin D, Bailleul B (1993) Mis-splicing yields circular RNA molecules. FASEB J. 7: 155-160.

17. Bailleul B (1996) During in vivo maturation of eukaryotic nuclear mRNA, splicing yields excised exon circles. Nucl. Acids Res. 24: 1015-1019.

18. Zaphiropoulos PG (1996) Circular RNAs from transcripts of the rat cytochrome P450 2C24 gene: correlation with exon skipping. Proc. Natl. Acad. Sci. U.S.A. 93: 6536-6541.

19. Capel B, Swain A, Nicolis S, et al. (1993) Circular transcripts of the testis-determining gene Sry in adult mouse testis. Cell 73: 1019-1030.

20. Jackson-Grusby L, Kuo A, Leder P (1992) A variant limb deformity transcript expressed in the embryonic mouse limb defines a novel formin. Genes Dev. 6: 29-37.

21. Sambrook J, Fritsch EF, Maniatis T (eds). (1989) Molecular Cloning: A Laboratory Manual, 2nd ed. Cold Spring Harbor Laboratory Press, Cold Spring Harbor, NY.

22. Melton DA, Krieg PA, Rebagliati MR, Maniatis T, Zinn K, Green MR (1984) Efficient in vitro synthesis of biologically active RNA and RNA hybridiza- tion probes from plasmids containing a bacteriophage SP6 promoter. Nucl. Acids Res. 12: 70357056.

23. Tybulewicz VL, Crawford CE, Jackson PK, Bronson RT, Mulligan RC (1991) Neonatal lethality and lymphopenia in mice with a homozygous disruption of the c-abl proto-oncogene. Cell 65: 1153-1163.

24. Deng C, Wynshaw-Boris A, Zhou F, Kuo A, Leder $P$ (1996) Fibroblast growth factor receptor 3 is a negative regulator of bone growth. Cell 84: 911921.

25. Deng C-X, Wynshaw-Boris A, Shen MM, Daugherty C, Ornitz DM, Leder P (1994) Murine FGFR-1 is required for early postimplantation growth and axial organization. Genes Dev. 8: 3045-3057.

26. Deng $C$, Zhang $P$, Harper JW, Elledge SJ, Leder $P$ (1995) Mice lacking p21 $1^{\text {CIPI/WAFI }}$ undergo normal development, but are defective in Gl checkpoint control. Cell 82: 675-684.

27. Chan DC, Leder P (1996) Genetic evidence that formins function within the nucleus. J. Biol. Chem. 271: 23472-23477.

28. Harlow E, Lane D (1988) Antibodies: A Laboratory Manual. Cold Spring Harbor Laboratory Press, Cold Spring Harbor, NY. 